\title{
Intermédialités
}

Histoire et théorie des arts, des lettres et des techniques

Intermediality

History and Theory of the Arts, Literature and Technologies

\section{Présentation : « au fil des oeuvres »}

\section{Maïté Snauwaert et Bertrand Gervais}

Numéro 7, printemps 2006

Filer (Sophie Calle)

Shadowing (Sophie Calle)

URI : https://id.erudit.org/iderudit/1005514ar

DOI : https://doi.org/10.7202/1005514ar

Aller au sommaire du numéro

Éditeur(s)

Centre de recherche sur l'intermédialité

ISSN

1705-8546 (imprimé)

1920-3136 (numérique)

Découvrir la revue

Citer ce document

Snauwaert, M. \& Gervais, B. (2006). Présentation : « au fil des oeuvres ».

Intermédialités / Intermediality, (7), 9-16. https://doi.org/10.7202/1005514ar d'utilisation que vous pouvez consulter en ligne.

https://apropos.erudit.org/fr/usagers/politique-dutilisation/ 


\title{
Présentation : «au fil des œuvres»
}

\author{
MAÏTÉ SNAUWAERT ET BERTRAND GERVAIS
}

«L'espace de notre vie n'est ni continu, ni infini, ni homogène, ni isotrope. Mais sait-on précisément où il se brise, où il se courbe, où il se déconnecte et où il se rassemble? On sent confusément des fissures, des hiatus, des points de friction, on a parfois la vague impression que ça se coince quelque part, ou que ça éclate, ou que ça se cogne. Nous cherchons rarement à en savoir davantage et le plus souvent nous passons d'un endroit à l'autre, d'un espace à l'autre sans songer à mesurer, à prendre en charge, à prendre en compte ces laps d'espace'. »

"Je me vois aux portes d'un labyrinthe, prête à me perdre dans la ville et dans

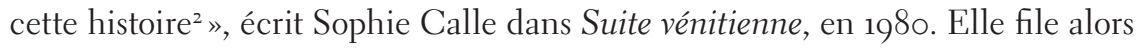
dans Venise un parfait inconnu, au motif que le destin a placé celui-ci deux fois sur son chemin. Moins fortuite, La filature, qui prend place l'année suivante, a été commanditée par sa mère auprès d'un détective privé, à la demande de l'artiste...

Filer Sophie Calle, c'est obéir au mouvement dont son œuvre donne l'impulsion, selon la réciprocité qui lui fait sans cesse échanger les positions d'objet et de sujet. C'est, surtout, poursuivre la trace de cette fileuse dans ses installations insolites ou les versions successives de ses projets, à travers ses autobiographies ou dans les interviews qu'elle fait de tiers anonymes. Filer, ce peut être suivre à la trace ou marcher vite et droit, s'envoler (à tire -d'aile) ou disparaître (à l'anglaise),

1. George Perec, Espèces d'espaces, Paris, Éditions Galilée, coll. «Espace critique », 1974, page de rabat.

2. Sophie Calle, «Suite vénitienne», dans À suivre..., Doubles-jeux (livre IV), Arles, Actes Sud, 1998, p. 44 . 
dessiner (un trait) ou décliner une image littéraire (la métaphore), voire s’évader, s'absenter, s'évanouir dans la nature...

Être aux portes d'un labyrinthe à la fois urbain et narratif. Cette situation est programmatique de l'une des orientations principales de l'œuvre de Sophie Calle: elle fait osciller le je entre les méandres d'un lieu concret et une profondeur narrative imaginaire, toujours au seuil d'une aventure nouvelle... Filer: le verbe illustre bien le travail d'une artiste aux multiples visages et la texture d'une œuvre protéiforme qui ne donne rien pour définitif, mais se réinvente sans cesse, tels ces êtres hybrides qu'on cache au centre de prisons labyrinthiques. Johnnie Gratton, l'un des premiers à avoir analysé l'œuvre de Calle, remarque :

Calle's enthusiasm for demonstrating the permutability of her previously published or exhibited phototextual sequences indicates clearly that, for her, as for numerous other contemporary artists (and, increasingly, writers), there is no such thing as a definitive, unproblematically date-bound, realisation of a given project ${ }^{3}$.

Son œuvre a une composante littéraire importante, qui l'inscrit dans la lignée des Perec et autres oulipiens pour le principe ludique d'une écriture à contraintes. Mais elle relève surtout fortement du champ plastique et du domaine des relations intermédiatiques, par la présence importante du photographique dans son travail, l'investissement de lieux, la mise en espace de personnes et d'objets dans des installations insolites, sans oublier l'apport vidéographique (Double Blind / No Sex Last Night [1992], Unfinished, [2003]). Cette pluralité de médias fait d'elle une artiste de la performance, tandis que la dimension narrative de son œuvre, alliée à un art de l'improvisé et du quotidien liant la réalité et l'imaginaire, la rapproche de Christian Boltanski, de Didier Bay ou de Jean Le Gac. On songe aussi à Alain Fleischer et à Denis Roche, pour la mixité de ses récits photographiques; ou à Cindy Sherman, à Annette Messager, à Jürgen Klauke, pour le travail de l'autoportrait, de l'autobiographie et, plus largement, de la mise en scène du moi. L'intermédialité de son œuvre joue également, au plan des relations qu'elle fait entretenir par l'art, avec la vie, et, à travers l'éventail de récits intimes aux provenances multiples, avec la sphère sociale.

«J'ai demandé » et «je fus invitée » sont les formules initiales récurrentes de nombreux projets, fondant la dynamique d'une grande partie du processus artistique de Calle, dans lequel l'influence voire la participation de tiers sont hautement

3. Johnnie Gratton, «Experiment and Experience in the Phototextual Projects of Sophie Calle», dans Gil Rye and Michael Worton (dirs.), Women's Writing in Contemporary France. New Writers, New Literatures in the 1990s, Manchester University Press, 2002 , p. 161. 
recherchées en amont et souvent thématisées dans le corps des œuvres. Manière habile de ne jamais s'attribuer le mérite ni la responsabilité totale des réalisations, de médiatiser l'autorité de son unique signature. Qu'il s'agisse des identités successives endossées par le narrateur universel des différents recueils de textes; de l'utilisation par l'écrivain Paul Auster de son «personnage» d'artiste; du jeu qu'elle décide ensuite de jouer avec ce dernier dans une collaboration où la fiction devient une manière de procuration; du rapport à cet «homme au carnet » dont elle fait un feuilleton-portrait pour Libération et qui se venge en répliquant dans le même quotidien; ou encore de Bénédicte Vincens, gardienne de l'exposition Le temps, vite à Beaubourg, admiratrice notoire de l'artiste, qui disparaît une nuit, à la suite de l'incendie de son appartement, et dont Sophie Calle expose l'absence; chaque fois, cette mise en relation devient pour l'artiste une manière de vivre et de créer. Une éthique qui produit son esthétique.

«J'ai suivi » ou bien «j'ai été suivie» forment l'autre partie de cette dynamique relationnelle qui alimente ses œuvres. Celle-ci a été repérée par la plupart des auteurs critiques dans les dix dernières années. Régine Robin écrit ainsi, à propos de La filature de 1981: "Sophie Calle construit une image d'elle-même dans ses parcours de hasard. Ce sont des autoportraits par procuration, elle se constitue comme objet de recherche, objet d'analyse, objet muséal. Elle interroge l'identité sur une frontière ténue entre l'ultrabanal du quotidien et la sophistication de mises en scène de traces de traces: filature, rapport, photographies, légendes, mini-récits ${ }^{4}$. „ Aujourd'hui, le premier terme de cette série, la filature, peut également être mis au pluriel, un pluriel exponentiel comme les «traces de traces » évoquées par Régine Robin, qui souligne à juste titre que l'artiste «ne s'aborde que médiatiquement ${ }^{5}$ ». À propos de l'homme suivi par Calle dans Suite vénitienne, Jean Baudrillard remarque que «le fait qu'elle soit là et qu'elle le suive est une sorte de preuve qu'il ne va nulle part. Cela ôte à sa pérégrination à lui son sens propre; en fait, le sens qu'il croit, lui, pouvoir avoir dans les diverses directions où il va, lui est caché, il est secret, il est derrière, c'est Sophie qui le suit ${ }^{6}$. »

Christine Angot décline le principe de la filature dans l'homophonie de l'être et du suivre lorsqu'ils se conjuguent à la première personne: «je suis / un

4. Régine Robin, Le Golem de l'écriture. De l'autofiction au cybersoi, Montréal, XYZ éditeur, coll. «Théorie et littérature», 1997, p. 219. Le chapitre quatre s'intitule: «Être sans trace: Sophie Calle», p. 217-230.

5. Régine Robin, Le Golem de l'écriture, p. 223.

6. Jean Baudrillard, Marc Guillaume, Figures de l'altérité, Paris, Descartes, 1992, p. 122. 
homme » s'oppose dans la démarche de Calle à «je suiz-un homme ». Jennifer Fisher note que Sophie Calle poursuit aussi ses visiteurs en exploitant l'audioguide comme un constituant à part entière de l'exposition. Dans La visite guidée, une installation de 1994, ce dispositif produit une logique interruptive par laquelle Calle hante constamment son visiteur: In the manner that Michel de Certeau has described the capacity of memory to interrupt space, each station on Calle's guided tour fractures the museum's public exhibitions with private and autobiographical assertions ${ }^{8}$. Pour Jean-Ernest Joos, le travail de l'artiste fait écho à celui de Massimo Guerrera dans l'importance accordée à la relation et à la circulation: "Lorsqu'elle distribue des sourires, des cigarettes et des sandwiches dans les rues de New York, selon le hasard des rencontres, souvent forcées, elle démontre que la contingence de l'autre ne fait pas obstacle à la relation, mais peut la générer9. » De cet aspect relationnel encore, Johnnie Gratton relève que "l'anthropologie du don, telle que l'infléchit le travail photo-textuel de Calle, sert [...] à mobiliser l'image d'un sujet relationnel, c'est-à-dire d'un sujet toujours ouvert et vulnérable à l'autre, toujours provisoirement situé ${ }^{10}{ }$. La relation est au cœur de l'œuvre et de sa continuation.

Il se peut qu'elle la nourrisse aussi en amont. Philippe Piguet remarque des questions D'où venons-nous? Que sommes-nous? Où allons-nous?, que posait déjà Gauguin, que «ce qu'elles interrogent des notions d'origine, d'identité et de destinée - partant d'altérité - constitue la dynamique même de la démarche de Sophie Calle" ». De là, il dresse de l'artiste le portrait suivant:

Sophie Calle appartient à une génération qui s'est appliquée à digérer les différentes propositions des avant-gardes des années 1960 en cherchant à en opérer une forme syncrétique. Son art, qui multiplie les procédures d'investigation, emprunte ses modalités aux pratiques conceptuelles, performantes et narratives se nourrissant volontiers des exemples tant d'une histoire de la littérature que du cinéma ${ }^{12}$.

7. Christine Angot, «No sex», Beaux Arts Magazine, n ${ }^{\circ}$ 234, novembre 2003, p. $80-86$.

8. Jennifer Fisher, «Speeches of Display: The Museum Audioguides of Sophie Calle, Andrea Fraser and Janet Cardiff », Parachute, n ${ }^{\circ}$ 94, avril-mai-juin 1999, p. 27-28.

9. Jean-Ernest Joos, «Communauté et relations plurielles. Dialogue entre les philosophes et les artistes», Montréal, Parachute, nº 100, octobre 2000, p. 52.

10. Johnnie Gratton, «Poétique et pratique du recueil photo-textuel dans l'œuvre de Sophie Calle », dans Irène Langlet (dir.), Le recueil littéraire. Pratiques et théorie d'une forme, Rennes, Presses Universitaires de Rennes, coll. «Interférences », 2003, p. 131.

11. Philippe Piguet, «Sophie Calle: un personnage en quête de soi», Paris, L'œil, $\mathrm{n}^{\mathrm{o}} 552$, novembre 2003 , p. 68.

12. Philippe Piguet, «Sophie Calle: un personnage en quête de soi», p. 69. 
Et Maïté Vissault note, de son côté :

En réunissant ses aventures dans de petits livres reliés en coffret, l'artiste perturbe des travaux qui auraient pu sembler motivés par un simple jeu conceptuel, façon Land Art en milieu social, par son histoire personnelle et ses interprétations, laissant basculer l'objectivité du concept dans la subjectivité de la perception et de la narration ${ }^{13}$.

Chaque héritage possible de Calle est donc revisité, décliné, re-tissé à sa manière.

Suiveuse en série mais aussi «artiste "m’as-tu-vu” », comme la désigne Isabelle Décarie ${ }^{14}$, Sophie Calle demande à être suivie, pistée, filée, d'exposition en intervention médiatique, d'installation en livre, de vidéo en feuilleton journalistique... «Comme dans toute performance, écrit Jean-Paul Guichard, - car il s'agit d'une performance - les photos, les écrits, les situations, la pensée de l'artiste aussi bien que son corps et son histoire sont les matériaux de l'Euvre-SophieCalle $^{15}$. » Lorsqu'elle répond elle-même au questionnaire qu'elle a composé avec Grégoire Bouillier pour Les Inrockuptibles ${ }^{16}$, à la question : "Qu'avez-vous été capable de faire par amour? ", elle déclare: «M'en aller sans bruit». À «Rédigez votre épitaphe », elle réplique: «À suivre... ». Et à la dernière, « Sous quelle forme aimeriez-vous revenir?»: «L'Arlésienne». L'à suivre oscille donc entre suite et série, temps et espace, poursuite et entendement, selon un double sens dont les dispositifs, dans leur ironie plaisante, sont coutumiers. Car leur sphère d'agissement se situe sans cesse entre plasticité et intellectualité, l'une s'incarnant dans l'autre, celle-ci rendue accessible par celle-là, qui est à prendre en tant que souplesse, porosité, facilité à infiltrer le monde empirique. L'art a à charge, et à cœur chez Calle, de rendre sensible - là aussi aux deux sens du terme : à la fois tactile, ou préhensible physiquement, et porteur d'une charge affective - ce monde qui demeurerait informe sans son intervention.

13. Maïté Vissault, «Entre subjectif et distancié », ETC Montréal, n 51, sept.-oct.nov. 2000 , p. 72

14. Isabelle Décarie, «Aimer souffrir », Spirale, n 198, sept.-oct. 2004, p. 35.

15. Jean-Paul Guichard, «Poker menteur: de la photographie comme preuve de l'existence de Sophie Calle», Danièle Méaux, Jean-Bernard Vray (dirs.), Traces photographiques, traces autobiographiques, Saint-Étienne, Publications de l'Université de SaintÉtienne, coll. «Lire au présent», 2004, p. 80-81.

16. Les Inrockuptibles, Sophie Calle et Grégoire Bouillier rédacteurs en chef, Paris, 19 novembre 2003 . 
Les dédicaces mêmes de Calle témoignent de cette orientation de l'œuvre. Dans À suivre...: «À mon père, Robert Calle, qui m’a encouragée à suivre et à ma mère, Rachel Sindler, qui m’a fait suivre» (c'est elle qui souligne). Dans De l'obéissance: «À Jean-Michel Othoniel qui m’a mise sur la piste...» Quant à LEErouv de Jérusalem, il rappelle que les erouvim «consistent en fils (ou cordes) formant un mur imaginaire. Dans la plupart des cas, ces "frontières" sont créées en érigeant des poteaux et en les connectant ensemble par l'intermédiaire de filins en acier galvanisé17.» Dans l'un des Journaux intimes ${ }^{18}$ enfin, on peut lire cette lettre, signée du calembour «- Votre amie Calle -»:

$[\ldots]$ vous ne m’avez pas [...] totalement suivie car vous avez opté cette semaine pour le fort joli nom, je le reconnais, de Sophie Caille. Je n'ai aucune antipathie pour l'oiseau en question, mais, voyez-vous, avec le temps, je m'étais habituée à mon nom, synonyme en espagnol de rue, donc d'errance... Mon prénom signifiant en grec: Sagesse, cela donnait «sagesse de la rue» et non «sage caille».

Et, à la page suivante, ce collage de publicité, peut-être réelle, portant le slogan «Sortez votre Sophie»:

Pratique... peu encombrante

dans votre sac, votre poche

toujours à votre disposition :

dans l'auto, la promenade, le voyage,

vos courses, etc...

Sa légèreté vous la fera oublier.

Il semble que l'artiste ait fait siennes, et celles de son œuvre, ces caractéristiques.

Filer, par sa signification à mi-chemin du littéraire et du plastique, fait résonner l'habileté technique comme la ruse imaginative. Le verbe nous a donc semblé propice à rendre compte de la multiplicité d'une pratique aussi singulière, et à «infinitiser» celle-ci. Les articles réunis dans ce dossier se prêtent, chacun selon sa ligne, à ce jeu.

Maïté Snauwaert suit d'abord le fil parfois rouge du récit à travers la systématicité de sa ligne de composition et la manière qu'il a de s'agencer par images. Les différentes acceptions de l'anecdote que le travail photolittéraire de Calle réalise sont visitées, et mises en relation avec la plasticité notamment poétique qui

17. Sophie Calle, L'Erouv de Jérusalem, Arles, Actes Sud, 1996, 2002, p. 8 et première page de rabat.

18. Sophie Calle, Journaux intimes (1978-1992), dans M'as-tu vue, catalogue de l'exposition, Christine Macel (éd.), Paris, Éditions du Centre Pompidou, Éditions Xavier Barral, 2003, p. 41-60. 
participe de leur précipitation en histoires, celles-ci à la fois éminemment ponctuelles et circulatoires, et gravées dans la pierre par l'assemblage du recueil.

Cécile Camart trace dans les œuvres récentes « les contours variables d'une place vacante » qui serait celle à la fois de l'identification du spectateur et d'une absence constitutive, dans des dispositifs qui sont d'abord des mises en espace avant que d'être des mises en livre, capables par conséquent de concrétiser la disparition, de donner au deuil une forme sensible, et si possible visible.

Bertrand Gervais piste le principe des «fantômes », ces planches que l'on met à la place des volumes sortis dans les bibliothèques et qui portent le nom de l'emprunteur, au sein du travail analogue de Calle dans L'absence. L'œuvre consiste alors à produire un simulacre collectif en lieu et place d'un tableau manquant, et les dispositifs de l'artiste mettent en scène l'emplacement de la hantise, qui poursuit gardiens et bientôt visiteurs ou lecteurs, cependant que les toiles elles-mêmes se souviennent de la présence de leurs modèles.

Perin Emel Yavuz, à travers le motif de la grille moderniste et dans la perspective historique et théorique du Narrative Art, étudie les premières filatures de l'artiste et leur rapport ambivalent au réel et à la fiction. Elle montre que la dimension narrative est déterminante de la mise en ouvre et de son caractère à la fois artistique et unifiant, en ceci notamment qu'elle convertit le temps empirique en récit. «Je suis pour suivre», une des phrases de Calle évoquée, ouvre à l'ambiguïté d'une démarche à la première personne qui, entre dilettantisme et artisticité, entre gratuité et engagement, fait œuvre.

Cybelle McFadden Wilkens examine le jeu de piste qui conduit Sophie Calle et Greg Shephard à travers les États-Unis dans la vidéo No Sex Last Night (1992). Le road trip les fait sans cesse reporter la confrontation et perpétuer l'évitement à travers l'outil interposé de leurs caméras respectives. Regards croisés pour un côte à côte plutôt qu'un face-à-face, qui aboutissent à une mise en question de la dimension auctoriale du travail de Sophie Calle. Alors qu'elle délègue régulièrement à des tiers une part de la responsabilité de son œuvre, son rapport aux autres et en particulier aux hommes semble demeurer toujours sous son contrôle.

Catherine Mavrikakis, dans le registre de l'invisible comme dans celui d'une forme de collaboration, convoque le photographe et auteur Hervé Guibert, alter ego des rendez-vous manqués, rencontré dans l'aveuglement commun qui les avait conduits, chacun à sa manière et de son côté, à travailler avec des aveugles, et que Calle poursuivit de ses assauts avant de le manquer définitivement, le jour de sa disparition. Plus qu'une dimension plastique, cette impossible coïncidence fait résonner l'accent nostalgique d'un conflit temporel: "Au moment où il se manifeste, le hasard a toujours déjà eu lieu dans le sentiment de sa prémonition. » 
Magali Nachtergael évoque cette esthétique de l'absence qui affecte non seulement l'œuvre mais encore la personne - le personnage? - de l'artiste, justifiant l'exploitation du photographique comme médium de la rétention, cependant que continue sa survivance face aux disparitions des autres. Elle nous rappelle que " "shadowing", en français, ce serait l'art de filer à l'anglaise, ou de disparaître en douce», mais aussi «le préliminaire à la broderie», au double sens du cousu et du raconté, dans l'intervalle duquel s'inscrit de nouveau l'intermédialité de l'artiste.

Martine Delvaux explore les qualités sexuelle et spectrale de la photographie, cet embaumement du présent dont elle montre qu'il est une médiation troublante, en relation d'interpénétration avec celle du récit: ghosting of the medium is effected in these narrativized pictures and photographic tales. Son travail sur les clichés file la métaphore d'un procédé à la fois photographique et littéraire qui peut conduire à l'aveuglement. Il s'allie plus largement à une mise en évidence des stéréotypes des rôles sexués qui sous-tendent l'œuvre callienne, à travers Des histoires vraies et Double Blind en particulier. Enfin, le sens spécifique qu'elle donne à la fiction chez l'artiste dessine l'insaisissabilité de celle-ci à travers les travestissements qui, faussement, l'exposent.

Si l'artiste est une fileuse, elle invite donc également, depuis les filatures de ses débuts, à la filer. Le dossier visuel qu'elle nous fait l'honneur de réaliser pour ce numéro, qui reprend sous une forme légèrement différente, et dans une présentation inédite, Vingt ans après, confirme cette orientation et [en] renouvelle l'invitation. 